\title{
Green Cooperative Sensing Scheme in Heterogeneous Networks
}

\author{
Lifei Shen ${ }^{1}$, Jian Liu ${ }^{* 2}$, Xinxin Tan', Lei Wang ${ }^{2}$ \\ ${ }^{1}$ School of Machnical Electronic \& Information Engineering, China University of Mining \& Technology, \\ Beijing 100083, China \\ [e-mail: 27097636@qq.com] \\ ${ }^{2}$ School of Computer \& Communication Engineering, University of Science and Technology Beijing, \\ Beijing 100083, China \\ [e-mail: jianliusdu@gmail.com; jennytanxx@163.com; 945295351@qq.com] \\ *Corresponding author: Jian Liu
}

Received January 27, 2017; revised April 14, 2017; revised August 22, 2017; accepted September 28, 2017; published February 28, 2018

\begin{abstract}
Cognitive radio technology is still the key technology of future mobile communication systems. Previous studies have focused on improving spectrum utilization and less energy consumption. In this paper, we propose an Overhead Reduced Scheme (ORS) for green cooperative spectrum sensing. Compared to traditional cooperative sensing scheme, ORS scheme divides the sensing time into three time slots and selects the best multi-mode user to report decisions. In consideration of reporting channel deviation, we derive closed-form expressions for detection probability and false alarm probability of ORS scheme based on Rayleigh fading channel. Simulation results show that ORS scheme can improve the perception accuracy while reducing the perceived delay and energy consumption in the process of perception, so as to realize the green communication.
\end{abstract}

Keywords: Geen communication, heterogeneous network, cooperative sensing, overhead saving, cognitive radio

This work is supported by National Major Project (No. 2015ZX03001013-002), Beijing Science and Technology Project (No. Z161100003016012) 


\section{Introduction}

Multi-radio access technology (multi-RAT) heterogeneous network has the advantages of large capacity, reduced interference and high spectrum utilization, which are the key requirements for modern communication networks [1-3]. The use of cognitive radio (CR) technology in multi-RAT heterogeneous networks allows for sensing the address location and status information so as to appropriately select the appropriate combinations of network parameters and RAT, and also optimize a relay node and bandwidth selection. This system can thus achieve maximum efficiency in a cost-effective manner [4-6].

In order to meet the emerging communication technology bandwidth requirements, early research focused on maximizing the discovery of unused spectrum without considering energy constraints. However, recent research has shifted to the seemingly contradictory problem of consuming less energy to obtain more spectrum. Authors of the literature [7] define the energy consumption per unit spectrum as opportunity cost. Based on this, we propose the cooperative sensing scheduling framework, which optimize awareness, reporting and channel conversion costs from the point of view of energy consumption [8-9]. In cognitive radio, unauthorized users continuously sense the licensed spectrum in order to detect the unused licensed spectrum by the primary users. In [10-11], the authors have proposed the collaborative cognitive radio network which utilized a finite number of samples for deciding the presence or absence of the primary signals. Because of this pivotal role, spectrum sensing is considered to be the most time-consuming and energy-consuming part of cognitive radio devices. Previous research works focused on the time overhead in the process spectrum sensing, In [12], the authors perform analysis of three major spectrum sensing techniques. In [13], authors design an optimal detection time by maximizing the throughput using an energy detection scheme. Literature [14-16] study the tradeoff between perceived time and energy efficiency in spectrum sensing. It is shown in [17-19] that the performance of a cognitive radio network can be improved by utilizing the double threshold detector for local detection. It is also confirmed that reliability of spectrum sensing can be improved in the CR by using multiple antennas. In [20], a multiple antenna based cooperative CR system with imperfect reporting channels is considered. In [21], the authors optimize detection performance using multiple antennas with two detectors.

Recently, due to the research trend of green communication, energy consumption in spectrum sensing has become one of the biggest challenges in academic research. J. Wei and X. Zhang propose the Distributed Spectrum Sensing (DSS) scheme based on cluster-forwarding. This scheme shows a significant decrease in total energy consumption while maintaining high detection accuracy. Literature [22-23] propose further improvements in spectrum sensing energy efficiency. [22] proposes time division energy saving (TDEE) aware technology, balancing energy consumption and spectral efficiency by studying heterogeneous and isomorphic networks. [24] provides a Converging Solutions for heterogeneous Mobile Networks, while in[25], the authors propose the selfish attacks and detection in cognitive radio. 
In this paper, we propose a green cooperative sensing scheme--ORS scheme, and focus on the perceptual scheduling problem of secondary users in heterogeneous networks. A SUE only has one RAT, while a multi-mode user equipment (MUE) is equipped with multiple RATs. The single-mode user equipments (SUEs) coexist with the MUEs in heterogeneous networks. By choosing the best cooperative MUE to send decision, we can increase the perceptual precision, while reducing the perceptual delay and the energy consumption of cooperative perception, thus realizing the green communication in the perceptual process.

\section{System Model}

We consider a heterogeneous wireless network consisting of cognitive user, multi-mode transmission user MUE (secondary collaboration users), main transmitting user PU(TX), main receiving user $\mathrm{PU}(\mathrm{RX})$, main base station $\mathrm{P}_{\mathrm{BS}}$, secondary base station $\mathrm{S}_{\mathrm{BS}}$ as depicted in Fig. 1. Each MUE equipped with multiple radio interfaces is capable to access multiple RATs simultaneously.

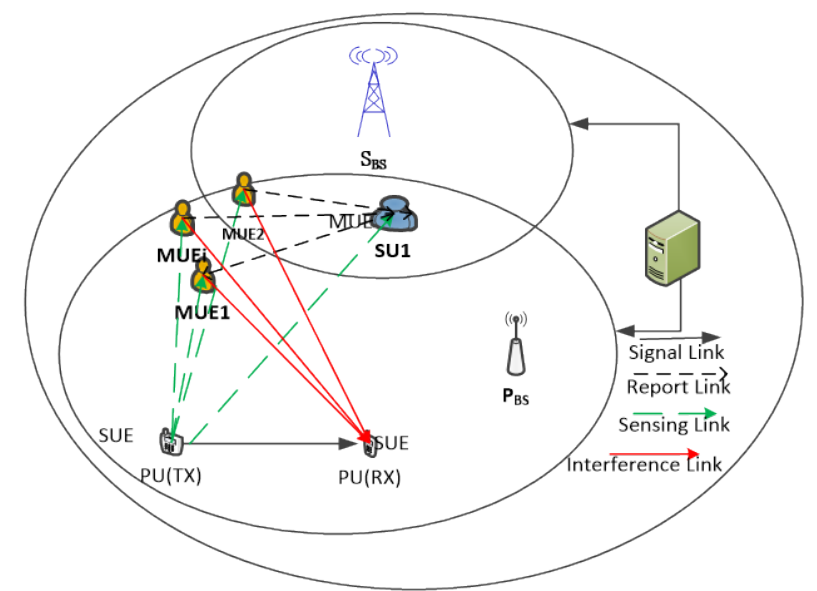

Fig. 1. System architecture

\subsection{Traditional Scheme}

Fig. 2 shows the time slot allocation in perception process of the traditional scheme. In order to reduce the interference, all secondary users send decision reports in different time slots. 


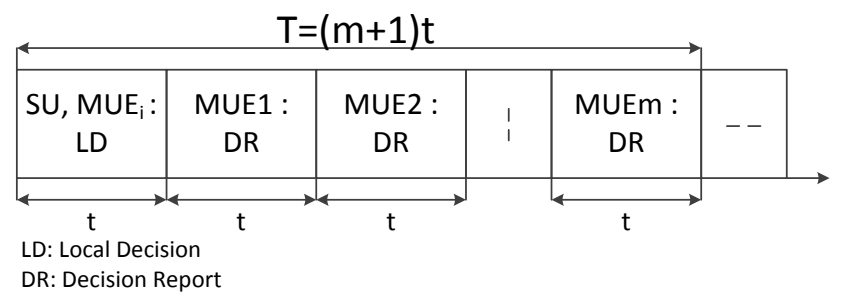

Fig. 2 Time slot of traditional scheme perception process

According to Fig. 1, the number of multi-mode users is $(m+1)$, consisting of a secondary user, m collaboration users, and all MUEs involved in the collaborative sensing process. SU executes local perception in the first sub period, and in the next $\mathrm{M}$ sub period, cognitive cooperative users report the decision to SU according to specific rules. SU then determines the final verdict according to all the local decisions and certain criteria. The perceived time of the system is given as $\mathrm{T}$, where $\mathrm{T}=(\mathrm{m}+1) \mathrm{t}$. Obviously, as the number of secondary collaboration users increases, the perceived time also increases. The transmission slot length is fixed, so the time for data transmission will be reduced. Assuming that average perceived energy consumption of each user is the same, the more users involved in the collaboration, the greater the perceived energy. According to Fig. 1, the local decision results reporting process will also interfere with the main transmission user, reducing the main transmission QoS.

\subsection{Improvement Scheme}

In order to improve the traditional cooperative sensing scheme, we propose an overhead saving scheme (ORS). ORS separates the local perceptions of the SU and MUEs and select the optimal MUE for decision reporting. Spectrum sensing and decision reporting use different the frequency bands in order to minimize the interference. i.e. cooperative users perform judgment reporting by Bluetooth frequency separating from the main user transmission frequency band. Fig. 3 shows the system architecture of ORS, while Fig. 4 illustrates the time slot allocation of ORS. 


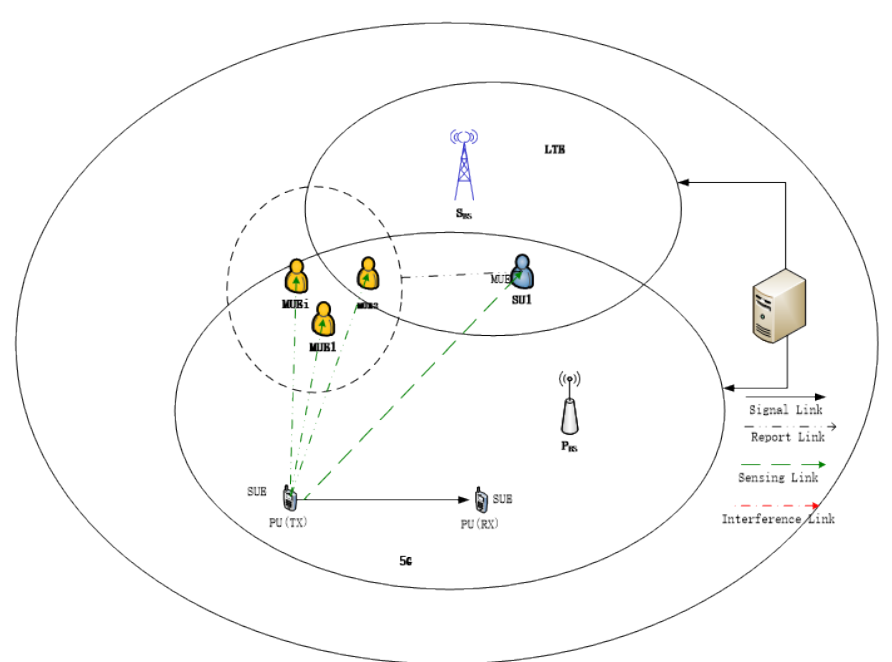

Fig. 3 System architecture of ORS

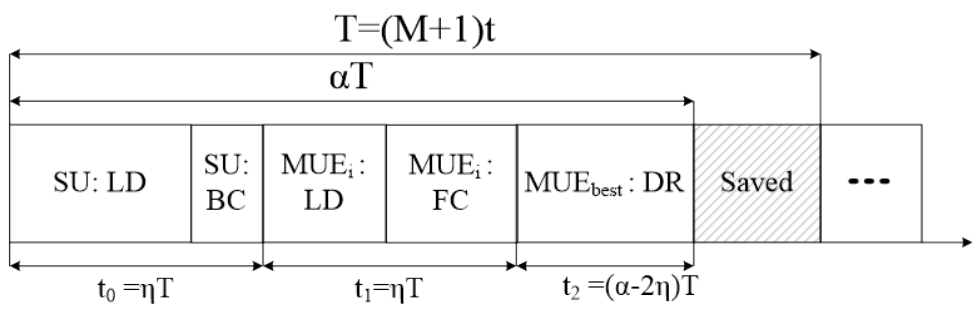

LD: Loca1 Detection; BC: Broad Cast; FC: Fusion Center; DR: Decision Report

Fig. 4 Time slot allocation of ORS

The execution of the ORS is shown in Fig. 5. The perception time is $\alpha T(0<\alpha \leq 1)$, and is divided into three slots: $\left\{\mathrm{t}_{0}, \mathrm{t}_{1}, \mathrm{t}_{2}\right\}, t_{0}=t_{1}=\eta T, t_{2}=(\alpha-2 \eta) T$. The concrete process is hereby described: in $t_{0}$, the SU performs local sensing to determine whether the primary transmission user exists. If so, the SU broadcasts to the secondary cooperating user and closes the process without temporarily accessing the spectrum of the primary user. If it does not exist, in a certain period of time, $\mathrm{MUE}_{\mathrm{i}}$ does not receive the broadcast information, then in $t_{1}, M_{i} E_{i}$ conducts local perception, and reports the results to the fusion center. Assuming $\mathrm{A}$ is defined as a set of $\mathrm{MUE}_{\mathrm{i}}$ that detects PU, if $\mathrm{A}$ is an empty set, the result of the cooperative detection shows that PU does not exist, and the collaboration will not report the decision. If $\mathrm{A}$ is not empty, we select the best collaboration and make decision report in $\mathrm{t}_{2}$. The best collaboration makes SU have the largest receiving SINR.

Different from the traditional cooperative sensing scheme, ORS takes advantage of multi-RAT access characteristics of cooperative users to perform judgment reporting by 
Bluetooth frequency separating from the main user transmission frequency band in order to minimize the interference.

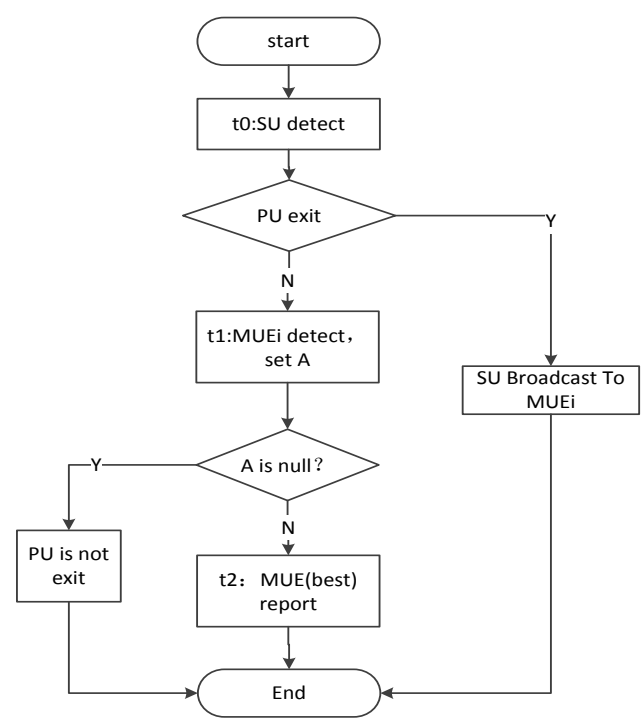

Fig. 5. Flow chart of ORS

\section{Theoretical Analysis}

\subsection{Perception Accuracy Analysis}

The local sensing of the ORS uses an energy detection scheme. The energy detector measures the received signal energy at a specific sensing time, and compares it with the established threshold to determine the two hypotheses [26].

$$
y_{i}(k)=\left\{\begin{array}{l}
n_{i}(k), H_{0} \\
h_{i} s(k)+n_{i}(k), H_{1}
\end{array}\right.
$$

$\mathrm{y}_{\mathrm{i}}(\mathrm{k})$ is received sample signal, $\mathrm{s}(\mathrm{k})$ and $\mathrm{n}_{\mathrm{i}}(\mathrm{k})$ are the signal and gauss white noise. $\mathrm{H}_{\mathrm{i}}$ is complex channel gain between the PU and the $\mathrm{MUE}_{\mathrm{i}}$. $\mathrm{s}(\mathrm{k})$ and $\mathrm{n}_{\mathrm{i}}(\mathrm{k})$ are independent identically distributed random process. $\mathrm{H}_{1}$ indicates that the primary transport user exists, while $\mathrm{H}_{0}$ indicates its absence. For $\mathrm{MUE}_{\mathrm{i}}$, the detection probability of energy detection can be expressed as [27]:

$$
\begin{aligned}
P_{d_{i}} & =\operatorname{Pr}\left\{\bar{H}_{1} \mid H_{1}\right\} \\
& =\mathrm{e}^{-\frac{\varepsilon_{i}}{2}} \sum_{k=0}^{\mu_{i}-2} \frac{1}{k !}\left(\frac{\varepsilon_{i}}{2}\right)^{k}+\left(\frac{1+\bar{\gamma}_{i}}{\bar{\gamma}_{i}}\right)^{\mu_{i}-1}\left[\mathrm{e}^{-\frac{\varepsilon_{i}}{2\left(1+\bar{\gamma}_{i}\right)}}-\mathrm{e}^{-\frac{\varepsilon_{i}}{2}} \sum_{k=0}^{\mu_{i}-2} \frac{1}{k !}\left(\frac{\varepsilon_{i} \bar{\gamma}_{i}}{2\left(1+\bar{\gamma}_{i}\right)}\right)^{k}\right] \\
& =\phi_{d}\left(\mu_{i}, \bar{\gamma}_{i}, \varepsilon_{i}\right)
\end{aligned}
$$


The false alarm probability of energy detection is:

$$
\begin{aligned}
P_{f_{i}} & =\operatorname{Pr}\left\{\bar{H}_{1} \mid H_{0}\right\} \\
& =\Gamma\left(\mu_{i}, \frac{\varepsilon_{i}}{2}\right) / \Gamma\left(\mu_{i}\right)
\end{aligned}
$$

$\mathrm{H}_{1}$ represents the judgment of the main transmission users' existence. $\mu_{i}$ indicates the time domain bandwidth product of energy detector and $\varepsilon_{i}$ indicates the energy threshold. $\gamma_{p}=E_{p} / \sigma_{n}^{2}$ is the signal power and noise power ratio of the main transmission users. For the convenience of future analysis, we use $\phi_{d}\left(\mu_{i}, \bar{\gamma}_{i}, \varepsilon_{i}\right)$ to express the local detection probability function and $\phi_{f}\left(\mu_{i}, \varepsilon_{i}\right)$ to express the local false alarm probability function [28]. For ORS, this paper uses $\lambda(0<\lambda<1)$ as the assumption of local false alarm probability and assumes the false alarm probability of cooperative users are equal. $\lambda_{0}$ is used to represent the overall false alarm probability. Thus, the energy threshold can be deduced as $\varepsilon_{i}=\phi_{f}^{-1}\left(u_{i}, \lambda\right)$ [29], where $\phi_{f}^{-1}$ is the inverse function of $\phi_{f}$. According to Eq. (2) and Eq. (3), in $t_{0}$, the local detection probability and false alarm probability of SU is:

$$
\begin{gathered}
P_{d}^{0}=\phi_{d}\left(\mu_{S U}^{O R S}, \bar{\gamma}_{S U}^{O R S}, \varepsilon_{S U}^{O R S}\right) \\
P_{f}{ }^{0}=\phi_{f}\left(\mu_{S U}^{O R S}, \varepsilon_{S U}^{O R S}\right)=\lambda
\end{gathered}
$$

$\mu_{S U}^{O R S}=t_{0} W_{e}=\eta T W_{e}, W_{e}$ is system bandwidth of energy detector, $\mathrm{T}$ is duration of the entire perceptual process, $\lambda$ is local false alarm probability, $\bar{\gamma}_{S U}^{O R S}=2 \mu_{S U}^{O R S} \gamma_{P U} \sigma_{P U, S U}^{2}$, and $\varepsilon_{S U}^{O R S}=\phi_{f}^{-1}\left(\mu_{S U}^{O R S}, \lambda\right)$.

$M U E_{i}$ assists $S U$ with spectrum sensing in time slots $t_{1}$, and energy detection technology is taken. The local detection probability and local false alarm probability of $\mathrm{MUE}_{\mathrm{i}}$ are:

$$
\begin{gathered}
P_{d_{i}}^{\text {ORS }}=\phi_{d}\left(\mu_{i}^{O R S}, \bar{\gamma}_{i}^{O R S}, \varepsilon_{i}^{O R S}\right) \\
P_{f_{i}}{ }^{\text {ORS }}=\phi_{f}\left(\mu_{i}^{O R S}, \varepsilon_{i}^{O R S}\right)
\end{gathered}
$$

where $\bar{\gamma}_{i}^{\text {ORS }}=2 \mu_{i}^{\text {ORS }} \gamma_{P U} \sigma_{P U, M U E_{i}}^{2}$.

In $t_{2}$, collaboration users who can maximize the receiver SINR in set A are selected to report results. At this point, the other MUEs listen to the best user's report. If the report is intercepted, no further reporting is performed. Assuming that $\mathrm{MUE}_{\mathrm{i}}$ is the best cooperative user, then the received signal of $S U$ in $t_{2}$ period can be expressed as:

$$
y_{S U, i}=\sqrt{E_{i}} h_{i, S U} x_{i}+\theta \sqrt{E_{P U}} h_{P U, S U} x_{P}+n_{S}
$$


MUE transmits a signal $x_{i}$ to its destination with power $E_{i} . h_{i, S U}$ represents the channel gain between $M_{U} E_{i}$ and SU. Similarly, PU transmits a signal $\mathrm{X}_{\mathrm{P}}$ to its destination with power $\mathrm{E}_{\mathrm{PU}}$. $\mathrm{H}_{\mathrm{PU}, \mathrm{SU}}$ represents the channel gain between $\mathrm{PU}$ and $\mathrm{SU} . \mathrm{N}_{\mathrm{S}}$ represents the received signal of SU. The choice of best collaboration users can be carried out according to Eq. (9) [30]:

$$
M U E_{\text {best }}=\max _{i \in A}\left(\frac{\gamma_{i}\left|h_{i, S U}\right|^{2}}{\theta \gamma_{P U}\left|h_{P U, S U}\right|^{2}+1}\right)=\max _{i \in A}\left(\gamma_{i}\left|h_{i, S U}\right|^{2}\right)
$$

A is a set of secondary collaboration users that can detect the existence of the main transmission users. For the set $\mathrm{A}$, in the case $H_{0}$, the probability that SU can successfully decode the verdict results from MUE $\mathrm{E}_{\text {best }}$ is:

$$
\begin{aligned}
P D_{H_{0}} & =\operatorname{Pr}\left\{\max _{i \in A}\left(\gamma_{i}\left|h_{i, S U}\right|^{2}\right) \geq \Delta\right\} \\
& =1-\prod_{i \in A}\left[1-\mathrm{e}^{-\frac{\Delta}{\gamma_{i} \sigma_{i, S U}^{2}}}\right]
\end{aligned}
$$

In the case $\mathrm{H}_{1}$, the probability that $S U$ can successfully decode the verdict results from $\mathrm{MUE}_{\text {best }}$ is:

$$
\begin{aligned}
P D_{H_{1}} & =\operatorname{Pr}\left\{\frac{\max _{i \in A}\left(\gamma_{i}\left|h_{i, S U}\right|^{2}\right)}{\gamma_{P}\left|h_{P U, S U}\right|^{2}+1} \geq \Delta\right\} \\
& =-\sum_{n=1}^{2^{|A|}-1}(-1)^{|A(n)|} \frac{\mathrm{e}^{-\sum_{i \in A(n)} \frac{\Delta}{\gamma_{i} \sigma_{i, S U}^{2}}}}{1+\sum_{i \in A(n)} \frac{\Delta \gamma_{P U} \sigma_{P U, S U}^{2}}{\gamma_{i} \sigma_{i, S U}^{2}}}
\end{aligned}
$$

$\Delta=2^{1 /(\alpha-2 \eta) T W_{s}}-1$, and $W_{s}$ represents the channel bandwidth of a sensing channel, $A(n)$ represents the $\mathrm{n}$-th nonempty subset of $\mathrm{A}$.

Depending on the probability of successful decoding, the probability of a false alarm that $\mathrm{SU}$ receives in $\mathrm{t}_{2}$ is:

$$
P_{f}^{1}=\sum_{j=1}^{2^{m}-1}\left\{\left[\prod_{i \in A_{j}} P_{f_{i}}^{O R S} \prod_{i \in A_{j}}\left(1-P_{f_{i}}^{O R S}\right)\right] P D_{H_{0}}\right\}
$$

The probability of a detection decision that $S U$ receives in $t_{2}$ is: 


$$
P_{d}^{1}=\sum_{j=1}^{2^{m}-1}\left\{\left[\prod_{i \in A_{j}} P_{d_{i}}^{O R S} \prod_{i \in A_{j}}\left(1-P_{d_{i}}^{O R S}\right)\right] P D_{H_{1}}\right\}
$$

$A_{j}$ is the j-th nonempty subset of $\mathrm{A}, \overline{A_{j}}$ is a complementary set of $A_{j}$, and $\left|A_{j}\right|$ is the number of elements. Therefore, the overall false alarm probability under ORS is given as:

$$
P_{f}=P_{f}^{0}+\left(1-P_{f}^{0}\right) P_{f}^{1}
$$

The overall detection probability under ORS is:

$$
P_{d}=P_{d}^{0}+\left(1-P_{d}^{0}\right) P_{d}^{1}
$$

Make $\phi(\lambda)=P_{f}=\lambda_{0}$, and the local false alarm probability of ORS is:

$$
\lambda=\phi^{-1}\left(\lambda_{0}\right)
$$

$\phi^{-1}$ is the inverse function of $\phi$.

Since in the third period, we select users from the set A to maximize the received signal-to-noise ratio to perform the decision reporting, so there is no fear of random access to the reported channel quality problems. Although the performance of the best relay selection is better in theory, choosing the best user needs to know the state of all channels in order to make the best judgment. So applying this scheme in practice would require considerable attention to the complexity of implementation, not just system performance.

Compared with the traditional scheme, the local sensing time can be extended and the detection accuracy can be improved by adjusting the value of $t_{0}$. Since the ORS scheme is selected for reporting, it can reduce the value of the overall perception of time, where $\alpha$ can represent the overall reduced value. By adjusting the value of $\alpha$ and $\eta$, the balance between detection accuracy and time can be determined.

\subsection{Perceived Overhead Analysis}

\section{(1) Perception Time}

The perception time is defined as the time that secondary users perceive the presence of the primary transmission users and access the authorized spectrum. In traditional cooperative sensing scheme, ST is related to the number of secondary users participating in collaboration and the unit-aware slot length.

$$
S T_{0}=T=(m+1) t
$$

In ORS, the length of perception depends on whether $S U$ can perceive the presence of PU. If detected, the perception time is $\mathrm{t}_{0}=\eta \mathrm{T}$, and the probability is $\mathrm{P}_{\mathrm{d}}$. Otherwise, $\mathrm{t}_{0}, \mathrm{t}_{1}$, and $\mathrm{t}_{2}$ will all be used similar to the traditional scheme. Thus, the average perceived time of the ORS scheme is given as: 


$$
\begin{aligned}
& S T_{1}=P_{d} \eta T+\left(1-P_{d}\right) \alpha T \\
& =P_{d} \eta(m+1) t+\left(1-P_{d}\right) \alpha(m+1) t \\
& =(m+1) t\left(P_{d} \eta+\alpha-\alpha P_{d}\right)
\end{aligned}
$$

As is known $0<P_{d}<1,0<\eta<\frac{1}{2} \alpha, 0<\alpha<1$, so

$$
\begin{aligned}
& 0<1-P_{d}<1 \\
& 0<\alpha\left(1-P_{d}\right)<1 \\
& 0<P_{d} \eta<\frac{1}{2} \alpha P_{d} \\
& 0<\alpha\left(1-P_{d}\right)+P_{d} \eta<\alpha\left(1-P_{d}\right)+\frac{1}{2} \alpha P_{d}<1
\end{aligned}
$$

Therefore, in the time overhead, the proposed ORS is better than the traditional cooperative sensing scheme. If $P_{d} \rightarrow 1, S T_{1} \rightarrow(m+1) t \eta$, if $P_{d} \rightarrow 0, S T_{1} \rightarrow(m+1) t \alpha$. It shows that if the secondary users have high local detection probability, ORS can greatly reduce the perception of time. Even if the local detection probability of secondary users is low, the time perception of ORS is $\alpha(0<\alpha<1)$ percent of the traditional scheme, therefore, the superiority of the ORS can be proved.

\section{(2) Opportunity Cost}

\section{A. Traditional Scheme}

Perceived energy is expressed by $E_{S 0}$, then:

$$
E_{S 0}=\left(\sum_{i=1}^{m} P_{s i}+P_{s}\right) t=P_{s}(m+1) t=P_{s} T
$$

$P_{s i}$ is energy consumption for energy detection of $M U E_{i}, P_{s}$ is energy consumption for energy detection of SU, we assume that $P_{s i}=P_{S}$.

$E_{X 0}$ is used to indicate reporting energy, and each user's reporting energy is $P_{x}$ :

$$
E_{X 0}=\sum_{i=1}^{m} P_{x i} t=P_{x} T
$$

The opportunity cost of the traditional scheme, i.e. cumulative energy consumption:

$$
E_{T 0}=E_{s 0}+E_{X 0}=\left(P_{s}+P_{x}\right) T
$$

\section{B. ORS}

Perceived energy is expressed by $E_{S 1}$, then: 


$$
E_{S 1}=P_{s} t_{0}+\sum_{i=1}^{m} P_{s i} t_{1}=P_{s}(m+1) \eta T
$$

$E_{X 1}$ is used to indicate reporting energy:

$$
E_{X 1}=P_{x} t_{2}=P_{x}(\alpha-2 \eta) T
$$

The opportunity cost of ORS, i.e. cumulative energy consumption:

$$
E_{T 1}=E_{S 1}+E_{X 1}=P_{s}(m+1) \eta T+P_{x}(\alpha-2 \eta) T
$$

\section{Simulation Analysis}

In this section, we simulate the ORS. We consider a cognitive radio system with PUs (TX, $\mathrm{RX}$ ) and $\mathrm{m}$ CRs (MUE) for simulation. Moreover, assuming the channel is independent Rayleigh fading, $h_{i j}$ is channel coefficients from user $i$ to user $\mathrm{j}$, variance is $\sigma_{i, j}^{2}$, mean Gaussian white noise is 0 , and variance is $\sigma_{n}^{2}$. User's perceived energy is $\mathrm{P}_{\mathrm{s}}$, reporting energy is $P_{x}$ and reporting duration is $t_{x}=100$ us.

Fig. 6 shows the relationship between energy consumption and the proportion of ORS. The energy consumption of ORS system is calculated from Eq. (25). As can be seen from the Fig. 6, energy consumption will increase in the same perceptual scenarios by increasing the number of collaborative users. On the other hand, for the same cooperative users, the energy consumption of the ORS is much smaller than that of the traditional scheme, implying that our improved scheme achieves significant power saving for spectrum sensing compared with the traditional case.

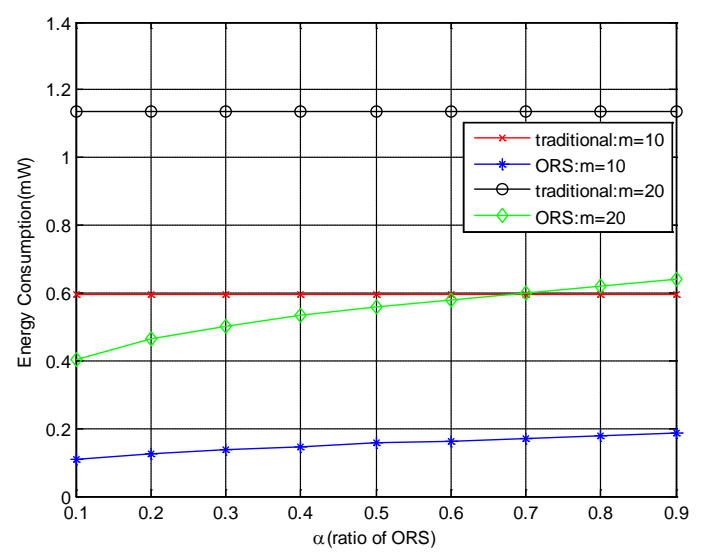

Fig. 6. Relationship between energy consumption and $\alpha$

Then, we plot the energy consumption versus local perception for the traditional and proposed schemes in Fig. 7. It is shown that the ORS scheme significantly reduce the energy consumption compared with traditional scheme. Assuming that the number of users 
involved in the collaboration is $\mathrm{m}=5$. when the value of $\alpha$ remains the same, energy consumption will increase along with the increase of $\eta$, which is because that local sensing energy is greater than energy consumption of the judgment report. So, the energy consumption of ORS scheme will increase remarkably with improving $\eta$. We can easily observe that energy consumption increases with larger $\alpha$. This is due to the fact that the total local perception radio increases as $\alpha$ increases. At the same time, the total judgment reporting ratio also increases, therefore, the larger $\alpha$ is, the greater the energy consumption.

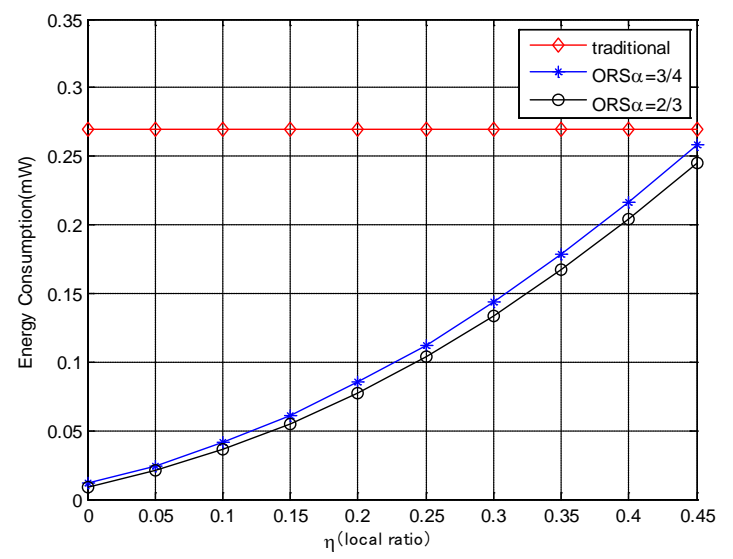

Fig. 7. Relationship between energy consumption and $\eta$

Fig. 8 illustrates the relationship between average perception time and the overall probability of false alarm. The average perceived time of the ORS scheme is calculated from Eq. (18), the ORS scheme has a great advantage over the conventional scheme in reducing the average perceptual time. For the ORS scheme, the higher the transmit SNR of the primary transmission user, the lower the energy consumption. That is due to the fact that local false alarm probability of secondary users being lower, and the perception time therefore reduces. When $\alpha_{P}$ is 10, comparing with scheme at $\alpha_{P t S U}=1$, ORS scheme at $\alpha_{P t S U}^{2}=0.6$ requires more average perceived time. When $\alpha_{P}$ remains the same value, the energy consumption will decrease as $\alpha_{P t S U}^{2}$ increases in proposed strategies due to the decrease of the local sensing energy of secondary users. 


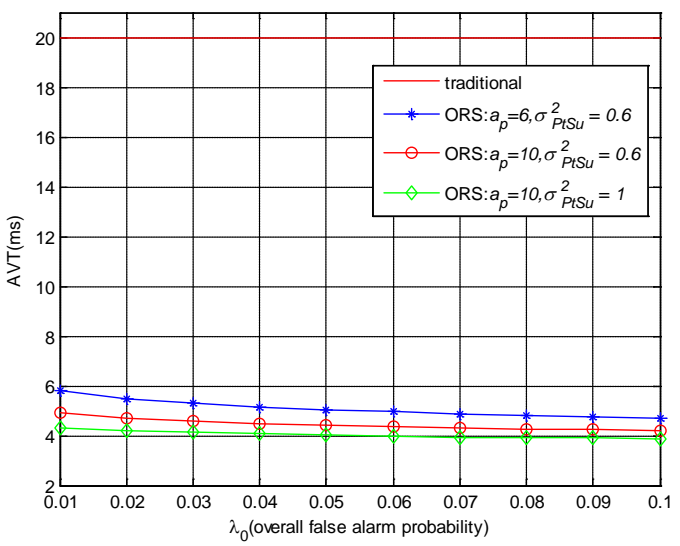

Fig. 8. Relationship between average perceived time and overall false alarm probability

Fig. 9 is the relationship between the energy consumption and the overall false alarm probability. It has the same trend as shown in Fig. 8. We can see that the energy consumption decrease as $\lambda_{0}$ grows since the local detection probability is improved.

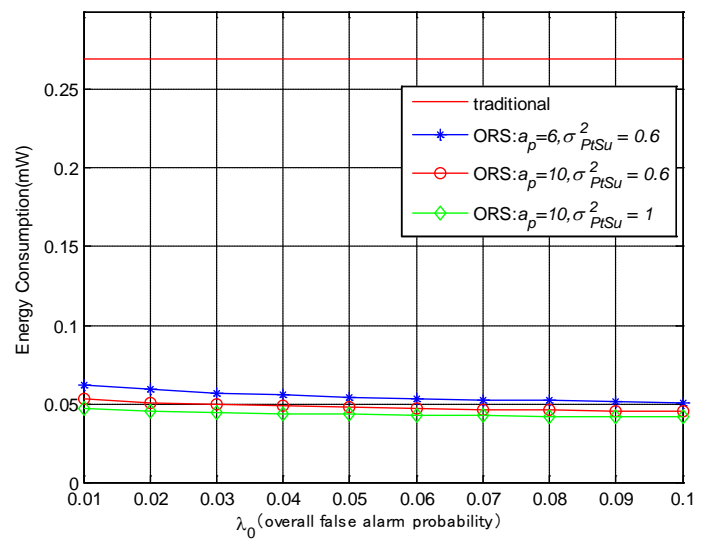

Fig. 9. Relationship between energy consumption and the overall false alarm probability

\section{Conclusion}

In this paper, we have proposed green cooperative sensing strategies based on efficient cost-saving strategy, called ORS scheme, to reduce the overall time and energy consumption. To achieve this, we propose a new time slot structure, which ORS scheme separates the local perceptions of the SU and MUEs, and select the optimal MUE for decision reporting. Based on these, we develop an effective scheme shown in the flow chart to select the optimal MUE for cooperative spectrum sensing. With considering the multi-path fading and the interference from PUs, we derived closed-form expressions of 
detection probabilities and false alarm probabilities over Rayleigh fading channels for ORS scheme. We also analyzed perception time and opportunity cost for the traditional and ORS scheme and compared the performance among them. Finally, numerical and simulation results confirm the effectiveness and improvement of proposed ORS scheme. It is shown that proposed strategies achieve lower sensing time and energy consumption but with higher implementation complexity than traditional case.

\section{References}

[1] Y. S. Soh, T. Q. S. Quek, M. Kountouris, H. Shin, "Energy Efficient Heterogeneous Cellular Networks,” IEEE J. Sel. Areas Commun, Vol. 31, No. 5, pp. 840-850, May 2013. Article (CrossRef Link)

[2] G. D. Yu, Y. H. Jiang, L. K. Xu, G. Y. Li, "Multi-Objective Energy-Efficient Resource Allocation for Multi-RAT Heterogeneous Networks,” IEEE J. Sel Areas Commun, Vol. 33, No. 10, pp. 2118-2127, Oct. 2015. Article (CrossRef Link)

[3] H. C. Wu, X. F. Tao, N. Li, J. Xu, "Secrecy Outage Probability in Multi-RAT Heterogeneous Networks,” IEEE Communication Letters, Vol. 20, No. 1, pp. 53-56, Jan. 2016. Article (CrossRef Link)

[4] C. L. I, C. Rowell, S. F. Han, et al. "Toward green and soft: a 5G perspective," IEEE Commun. Mag, Vol. 52, No. 2, pp. 66-73, Feb. 2014. Article (CrossRef Link)

[5] A. Celik, A. E. Kamal, "Green Cooperative Spectrum Sensing and Scheduling in Heterogeneous Cognitive Radio Networks," IEEE Transactions on Cognitive Communications and Networking, Vol. 2, No. 3, pp. 238-248, Sept. 2016. Article (CrossRef Link)

[6] Y. H. Choi, Y. Lee, J. M. Cioffi, "Optimization of Cooperative Inter-Operability in Heterogeneous Networks with Cognitive Ability,” IEEE Communications Letters, Vol. 15, No. 11, pp. 1178-1180, Nov. 2011. Article (CrossRef Link)

[7] C. G. Yang, J. Yue, M. Sheng, J. D. Li, "Tradeoff between energy-efficiency and spectral-efficiency by cooperative rate splitting," Journal of Communications and Networks, Vol. 16, No. 2, pp. 121-129, Apr. 2014. Article (CrossRef Link)

[8] A. Celik, A. E. Kamal, "More spectrum for less energy: Green cooperative sensing scheduling in CRNs,” IEEE International Conference on Communications, pp. 62-67, Sept. 2015. Article (CrossRef Link)

[9] M. Pirmoradian, O. Adigun, C. Politis, "An analytical evaluation of energy consumption in cooperative cognitive radio networks,” Building Sustainable Communities, pp. 1-6, Apr. 2013. Article (CrossRef Link)

[10] A. Singh, M. R. Bhatnagar, R. K. Mallik, "Threshold optimization of a finite sample-based cognitive radio network using energy detector," EURASIP Journal on Wireless Communications and Networking, Vol. 2013. Article (CrossRef Link)

[11] A. Singh, M. R. Bhatnagar, R. K. Mallik, "Threshold Optimization of Finite Sample Based Cognitive Radio Network,” National Conference on Communications (NCC), Feb. 2012. Article (CrossRef Link)

[12] A. Bagwari, B. Singh, "Comparative Performance Evaluation of Spectrum Sensing Techniques for Cognitive Radio Networks," IEEE International Conference on Computational Intelligence and Communication Networks, pp. 98-105, 2012. Article (CrossRef Link)

[13] S. J. Shellhammer, "Spectrum Sensing in IEEE 802.22," IAPR Wksp. Cognitive Info. Processing, 2008. Article(CrossRefLink) 
[14] Y. Pei, Y. Liang, K. C. Teh, K. H. Li, "Sensing-throughput tradeoff for cognitive radio networks: A multiple-channel scenario,” IEEE Trans, Vol. 7, No. 4, pp. 1326-1337, Apr. 2008. Article (CrossRef Link)

[15] J. Zhang, F. C. Zheng, X. Q. Gao, H. B. Zhu, "Sensing-energy efficiency tradeoff for cognitive radio networks,” IET Communications, Vol. 8, No. 18, pp. 3414-3423, Dec. 2014. Article (CrossRef Link)

[16] A. Celik, A. E. Kamal, "Multi-Objective Clustering Optimization for Multi-Channel Cooperative Spectrum Sensing in Heterogeneous Green CRNs,” IEEE Trans, Vol. 2, No. 2, pp. 150-161, Jun. 2016. Article (CrossRef Link)

[17] A. Bagvari, G. S. Tomar, S. Verma, "Cooperative Spectrum Sensing Based on Two-Stage Detectors With Multiple Energy Detectors and Adaptive Double Threshold in Cognitive Radio Networks,” Wireless Personal Communications, Vol. 70, No. 4, pp. 1-5, 2013. Article (CrossRef Link)

[18] A. Bagwari, G. S. Tomar, S. Verma, "Cooperative Spectrum Sensing Based on Two-Stage Detectors With Multiple Energy Detectors and Adaptive Double Threshold in Cognitive Radio Networks, " Canadian Journal of Electrical and Computer Engineering, Vol. 36, No. 4, pp. 172-180, 2014. Article (CrossRef Link)

[19] A. Bagwari, G. S. Tomar, "Two-Stage Detectors with Multiple Energy Detectors and Adaptive Double Threshold in Cognitive Radio Networks," International Journal of Distributed Sensor Networks, Vol. 9, No. 8, pp. 1-8, 2013. Article (CrossRef Link)

[20] A. Singh, M. R. Bhatnagar, R. K. Mallik, "Cooperative Spectrum Sensing in Multiple Antenna Based Cognitive Radio Network Using an Improved Energy Detector," IEEE Communications Letters, Vol. 16, No. 1, pp. 64-67, 2012. Article (CrossRef Link)

[21] J. Kanti, G. S. Tomar, "Improved sensing detector for wireless regional area networks," the International Journal of Cogent Engineering, Vol. 4, No. 1, Jan. 2017. Article (CrossRef Link)

[22] J. Wei, X. Zhang, "Energy-Efficient Distributed Spectrum Sensing for Wireless Cognitive Radio Networks," INFOCOM, IEEE Conference on Computer Communications Workshops, Mar. 2010. Article (CrossRef Link)

[23] G. Sharma, R. Sharma, "A review on recent advances in spectrum sensing, energy efficiency and security threats in cognitive radio network," Microwave, Optical and Communication Engineering International Conference, pp. 114-117, Dec. 2015. Article (CrossRef Link)

[24] M Jo, T Maksymyuk, R L Batista, "Survey of Converging Solutions for heterogeneous Mobile Networks,” IEEE Wireless Communications, Vol. 21, No. 8, pp. 54-62, Dec. 2014. Article (CrossRef Link)

[25] M Jo, L Han, D. kim, "Selfish Attacks and Detections in Cognitive Radio Ad-hoc Networks," IEEE Network, Vol. 27, No. 3, pp. 46-50, Jun. 2013. Article (CrossRef Link)

[26]Y. Liu, S. Xie, Y. Zhang, et al., "Energy-efficient spectrum discovery for cognitive radio green networks,” Mobile networks and applications, Vol. 17, No. 1, pp. 64-74, 2012. Article (CrossRef Link)

[27] Z Dai, J. Liu, K. Long, "Selective-Reporting-Based Cooperative Spectrum Sensing Strategies for Cognitive Radio Networks,” IEEE Trans, Vol. 64, No. 7, pp. 3043-3055, 2015. Article (CrossRef Link)

[28]C. Yang, J. Yue, M. Sheng, et al. "Tradeoff between energy-efficiency and spectral-efficiency by cooperative rate splitting," Journal of Communications and Networks, Vol. 16, No. 2, pp. 121-129, 2014. Article (CrossRef Link)

[29] P. Lin, J. Jia, Q. Zhang, et al. "Dynamic spectrum sharing with multiple primary and secondary users,” IEEE Trans, Vol. 60, No. 4, pp. 1756-1765, 2011. Article (CrossRef Link) 
[30] Y. Chen, S. Zhang, S. Xu, et al. "Fundamental trade-offs on green wireless networks,” IEEE Commun. Mag, Vol. 49, No. 6, pp. 30-37, 2011. Article (CrossRef Link)

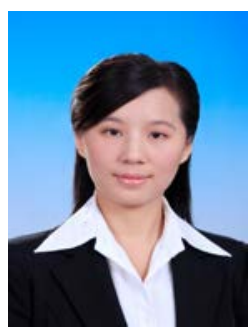

Lifei Shen was born in Hebei province, China. She got the Bachelor degree of mathematics and applied mathematics from Langfang Teachers University in 2007 and the Master degree of fundamental mathematics from China University of Mining \& Technology Beijing in 2011, respectively. Now she is Pursuing her PH. D. degree of control theory and control engineering in School of Machnical Electronic \& Information Engineering at China University of Mining \& Technology Beijing. Her research interests include cognitive radio, and next generation wireless communication.

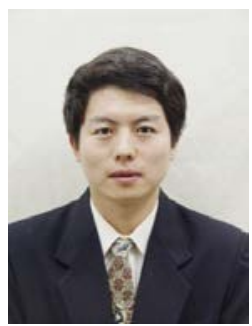

Jian Liu received his B.S. degree in Automatic Control Theory and Applications from Shandong University, China, in 2000, and the Ph.D. degree in School of Information Science and Engineering from Shandong University in 2008. He is currently an associate professor of University of Science and Technology (USTB), Beijing, China. His research interests include cognitive radio networks, mobile mesh networks, and LTE-A. He is an IEEE member since 2009.

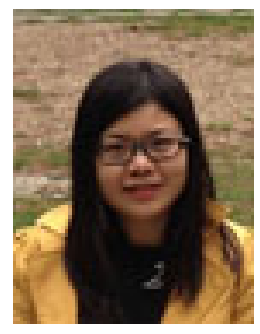

Xinxin Tan was born in Hubei Province, China. She got the Bachelor's degree of University of Science \& Technology Beijing (USTB) in 2014, and now she is studying for a master's degree at USTB. Her research field includes wireless communication and cognitive radio.

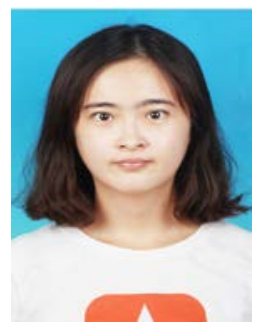

Lei Wang was born in Shandong province, China. She got the B.S. degree in Electronic Information of Science and Technology from Qingdao University of Science and Technology, China in 2015, and now she is studying for M.S. degree at University of Science and Technology Beijing. Her research interests include wireless communication and cognitive radio. 